Revista Eureka sobre Enseñanza y Divulgación de las Ciencias

ISSN: 1697-011X

revista.eureka@uca.es

Universidad de Cádiz

España

\title{
Revisión de experiencias sobre prácticas científicas en secuencias educativas de geología con trabajo de campo
}

Uskola, Araitz; Seijas, Nahia; Sanz, Josu

Revisión de experiencias sobre prácticas científicas en secuencias educativas de geología con trabajo de campo

Revista Eureka sobre Enseñanza y Divulgación de las Ciencias, vol. 19, núm. 1, 2022

Universidad de Cádiz, España

Disponible en: https://www.redalyc.org/articulo.oa?id=92068491016

DOI: https://doi.org/10.25267/Rev_Eureka_ensen_divulg_cienc.2022.v19.i1.1105 


\section{Revisión de experiencias sobre prácticas científicas en secuencias educativas de geología con trabajo de campo}

Scientific practices in geology educational sequences with field work. Review of experiences

Araitz Uskola

Departamento de Didáctica de la Matemática y de las

Ciencias Experimentales y Sociales, Facultad de Educación

de Bilbao. Universidad del País Vasco UPV/EHU. Leioa,

España

araitz.uskola@ehu.eus

iD https://orcid.org/0000-0003-0621-3085

Nabia Seijas

Departamento de Didáctica de la Matemática y de las

Ciencias Experimentales y Sociales, Facultad de Educación

de Bilbao. Universidad del País Vasco UPV/EHU. Leioa,

España

nahiaseijas@gmail.com

iD https://orcid.org/0000-0002-9922-7963

Josu Sanz

Departamento de Didáctica de la Matemática y de las

Ciencias Experimentales y Sociales, Facultad de Educación,

Filosofía y Antropología II. Universidad del País Vasco

UPV/EHU. Donostia - San Sebastián, España

josu.sanz@ehu.eus

iD https://orcid.org/0000-0002-1211-1598
DOI: https://doi.org/10.25267/

Rev_Eureka_ensen_divulg_cienc.2022.v19.i1.1105

Redalyc: https://www.redalyc.org/articulo.oa?

$\mathrm{id}=92068491016$
Recepción: 09 Junio 2021

Revisado: 24 Julio 2021

Aprobación: 30 Octubre 2021

\section{ReSUMEN:}

Para desarrollar la competencia científica, el alumnado tiene que apropiarse de la cultura científica, a través de las prácticas científicas. En la enseñanza de la geología el campo es también un contexto para la enculturación. Se ha estudiado muy poco la interrelación entre prácticas y trabajo de campo. En este trabajo se realiza una revisión de los artículos que incluyen salida de campo, publicados en diez revistas de enseñanza de las ciencias los últimos diez años. Se identificó la mención a operaciones relacionadas con las prácticas científicas en 20 artículos. La argumentación fue identificada en todos los grupos de edad, la indagación prácticamente solo en el ámbito universitario y la modelización apenas tuvo presencia. Se concluye que la salida jugó un papel en el desarrollo de las prácticas más allá de la recogida de datos. Se detectó la ausencia de operaciones fundamentales en las prácticas, por lo que se plantean algunas implicaciones educativas ilustradas con buenas prácticas identificadas.

Palabras clave: Competencia científica, Enseñanza de las ciencias, Prácticas científicas, Revisión sistemática, Trabajo de campo.

\section{ABstraCt:}

In order to develop scientific competence, students have to appropriate scientific culture through scientific practices. In geology teaching, the field is also a context for enculturation. The interrelation between practices and fieldwork has been scarcely studied. In this paper a review is made of articles that include field trips, published in ten science teaching journals over the last ten years. Mention of operations related to scientific practices was identified in 20 articles. Argumentation was identified in all age groups, 
inquiry practically only at university level and modelling was hardly present at all. It is concluded that fieldwork played a role in the development of practices beyond data collection. The absence of fundamental operations in the practices was detected, which leads to raise some educational implications illustrated with identified good practices.

KEYWORDS: Fieldwork, Science education, Scientific literacy, Scientific practices, Systematic review.

\section{INTRODUCCIÓN}

Los estudios sobre la enseñanza de ciencias en Europa (Osborne y Dillon 2008) y en España (COSCE 2011) constatan la necesidad de reformarla y convertir el aprendizaje de las ciencias, en consonancia con el marco del aprendizaje situado (Brown et al. 1989), en un proceso de enculturación, de apropiación de la cultura científica. El trabajo de campo y la incorporación de prácticas científicas pueden suponer una oportunidad única para practicar ciencias en este sentido. A nivel internacional, por ejemplo, en EEUU (NRC 2012), las reformas de la enseñanza de las ciencias incorporan en este momento el término prácticas científicas como uno de los ejes sobre los que pivota su propuesta.

En el aprendizaje de la geología se considera que la actividad práctica genuina por excelencia es el trabajo de campo (Pedrinaci 2012), y así las salidas son un contexto de referencia en la enculturación científica (Donaldson et al. 2020). En las salidas se abre a los estudiantes la posibilidad de analizar múltiples interpretaciones sobre lo ocurrido en el pasado, pueden mejorar el razonamiento causal y el pensamiento dinámico, además de conocer de primera mano hitos locales de interés geológico, e incluso relacionar estos con problemáticas sociales y ambientales.

Se han realizado varias revisiones sobre trabajos que incluyen salidas, tales como la de Rickinson et al. (2004), que examinaron 150 trabajos publicados entre 1993 y 2003, sobre el aprendizaje fuera del contexto escolar, y resaltaron el impacto positivo de las salidas en el ámbito afectivo. Behrendt y Franklin (2014) realizaron un análisis de los resultados de estudios que incluían todo tipo de salidas, concluyendo que todos señalaban la potencialidad de las salidas para motivar al alumnado, para favorecer que los estudiantes sean sujetos activos y puedan indagar, para comprender en más profundidad los conceptos, y para aumentar el interés por la ciencia. En una revisión más reciente, Aguilera (2018) realizó una descripción del país, disciplina científica, contexto, metodología y etapa de la salida en las publicaciones en WoS y Scopus de 2001 a 2017, señalando el potencial de las salidas de campo para la promoción de actitudes y el aprendizaje, así como los factores a tener en cuenta para aprovechar dicho potencial y no limitar su uso.

En geología las salidas de campo pueden tener un papel fundamental en el desarrollo de cada una de las tres prácticas científicas. Es de interés conocer si en las experiencias con prácticas científicas el papel otorgado a la salida se limita a la recolección de datos o a ilustrar lo trabajado en clase, o si por el contrario, se le está dando un papel más sustancial para la realización de prácticas científicas. En la literatura no se han hallado trabajos que aborden esta cuestión. Este estudio pretende contribuir a este conocimiento, dando respuesta a las siguientes preguntas de investigación:

1. ¿De qué manera se alude al desarrollo de prácticas científicas en las secuencias de enseñanza de la geología que incluyen salida de campo?

2. ¿En qué grado se utiliza la salida de campo respecto al aula para el desarrollo de operaciones relacionadas con las prácticas científicas en las experiencias de enseñanza de la geología que incluyen salida de campo y que desarrollan prácticas científicas?

3. ¿En qué grado se mencionan las operaciones relacionadas con la indagación, argumentación y modelización en las experiencias de enseñanza de la geología que incluyen salida de campo y que desarrollan prácticas científicas? 


\section{LAS SALIDAS DE CAMPO EN EL APRENDIZAJE DE LA GEOLOGÍA}

Uno de los objetivos de la enseñanza de las ciencias es comprender el mundo natural y los procesos y relaciones que ocurren en él. Ese mundo está fuera del aula, y por tanto el papel de las salidas de campo es insustituible (Pedrinaci 2012). Son muchos los potenciales beneficios que justifican el interés didáctico de las salidas al medio, y no deberían verse las salidas de campo como una mera extensión de lo que se aprende en clase, sino como un contexto educativo genuino, con metodologías y estrategias de enseñanza y aprendizaje propias (DeWitt y Storksdieck 2008).

Algunos de los beneficios de las salidas de campo para el aprendizaje de las ciencias son de corte actitudinal (Lavie y Tal 2017, Orion y Hofstein 1994). Es tal la impronta que en ocasiones dejan las salidas al medio natural que, por ejemplo, docentes en formación que han vivido experiencias positivas como estudiantes muestran una valoración muy alta sobre la importancia de las salidas como instrumento educativo (Costillo et al. 2014).

El trabajo de campo forma parte de la práctica y de la cultura de la ciencia geológica (Mogk y Goodwin 2012; Petcovic et al. 2014). Esto ocurre porque la geología es una ciencia histórica e interpretativa, que trata de explicar fenómenos ocurridos en el pasado a través de la interpretación de registros del presente (Frodeman 1995). Estos registros están fragmentados espacialmente y temporalmente, y se estructuran mediante representaciones visuales como mapas, cortes y columnas estratigráficas (Balliet et al. 2015), la mayoría de veces in situ, en el campo.

Es sobre el terreno donde el estudio y la interpretación de la geología adquieren la mayoría de su significado (van Loon 2008), ya que el estudiante está literalmente inmerso en el escenario real donde se materializan las representaciones mentales de los objetos y procesos geológicos. Muchas veces, la escala de los objetos de estudio es muy grande en relación al observador (kilométrica, por ejemplo). Cuando los estudiantes están en el campo, están inmersos espacialmente en las estructuras geológicas, una perspectiva única e irreproducible en el laboratorio (Mogk y Goodwin 2012). Fedesco y Cavin (2020) detectaron la importancia de este hecho en su estudio cualitativo sobre los beneficios de las salidas de campo en varios grados universitarios: los estudiantes de geología indicaban que el campo les permitió ver el "marco completo".

El campo es un recurso imprescindible en el desarrollo de las prácticas científicas en geología; pues es el sitio clave donde se recogen e interpretan la mayoría de los datos, que sirven para formular hipótesis y construir modelos, prácticas que pueden realizarse asimismo en las salidas (Balliet et al. 2015). Por último, los datos del campo también pueden ser empleados en argumentos, como evidencias para poner a prueba un modelo (Blanco-Anaya, Justi y Diaz de Bustamante 2017).

Que las prácticas científicas sean incluidas en las salidas de campo depende en gran medida del diseño de la secuencia didáctica que haga el docente. Así, una adecuada estrategia instruccional puede condicionar a que una salida se convierta en una práctica de investigación donde el estudiantado actúa como sujeto activo o bien sea simplemente la tradicional salida fuera del aula que no vaya más allá de la observación real de lo visto en clase (Behrendt y Franklin 2014; Carrier et al. 2013, Orion y Hofstein 1994). Es importante, por tanto, una adecuada formación y motivación de los docentes, ya que en muchas ocasiones estos admiten que las salidas que realizan distan mucho de la práctica investigativa que tenían en mente cuando las prepararon (Morcillo 1998, Midyan y Orion 2003).

\section{LAS PRÁCtiCAS CIENTíficAS EN EL APRENDIZAJE dE LA GEOLOGía}

Existe un amplio consenso en la comunidad experta en enseñanza de las ciencias sobre la necesidad de incorporar al ámbito educativo actividades más cercanas a las prácticas propias de la ciencia. Se ha adoptado el término prácticas científicas para designar a tales actividades (NRC 2012, Osborne 2014). Como indica Egger (2019) las Ciencias de la Tierra han ido algo más lentas que el resto en la implantación de este tipo 
de metodologías, pero cada vez más se encuentran buenos ejemplos de transformación del curriculum y de la práctica docente a este tipo de actividades de investigación escolar auténtica (Moss y Cervato 2016). Las prácticas científicas se pueden agrupar en tres, ya que se relacionan con el diseño y puesta en marcha de investigaciones, con el uso de pruebas y construcción de argumentos, y con la expresión, revisión y evaluación de modelos (Jiménez-Aleixandre y Crujeiras 2017).

\section{Indagación}

La indagación es un proceso para descubrir nuevas relaciones causales (Pedaste et al. 2015), donde el alumnado formula preguntas e hipótesis y las somete a prueba realizando experimentos y/o observaciones. Para que una actividad sea considerada de indagación debe cumplir dos condiciones: que incluya una pregunta investigable (Harlen 2014, Martí 2012, Sanmartí y Márquez 2012) y que los estudiantes analicen datos y utilicen evidencias para dar sentido o respuesta a un evento o fenómeno natural (Bell et al. 2010, en Ferrés et al. 2015). La indagación se desarrolla en diferentes fases, cuyo orden depende del contexto, y cuyo desarrollo implica varias operaciones (Harlen 2014, NRC 2012, Pedaste et al. 2015) relacionadas con la identificación y formulación de preguntas que guíen la investigación, la formulación de hipótesis y predicciones, la planificación de la investigación, y la recogida y análisis de datos. En las investigaciones del tipo de control de variables habrá además operaciones relacionadas con la identificación y control de variables, y en las de observación, operaciones de observación (Schalk et al. 2013). La propia naturaleza interpretativa de la geología, donde hay que considerar diferentes opciones, juega a favor de que los estudiantes puedan desarrollar la formulación de diversas hipótesis (Ault 1998).

\section{Argumentación}

La argumentación es la «capacidad de relacionar explicaciones y pruebas o, en otras palabras, de evaluar el conocimiento basándose en las pruebas disponibles» (Jiménez-Aleixandre 2010, p.11). Las operaciones que comporta esta práctica tienen que ver, por tanto, con la interpretación y uso de pruebas, con la formulación de conclusiones a partir de las mismas, con la elaboración de justificaciones, entendidas como elementos que conectan los datos con las conclusiones, y con la elaboración de refutaciones a los argumentos contrarios (Bargiela et al. 2018, Chang y Chiu 2008, Erduran et al.2004, Jiménez-Aleixandre 2010, Jiménez-Aleixandre y Crujeiras 2017, NRC 2012, Osborne et al.2016).

Trend (2009) indica que las ciencias de la tierra son propicias para la argumentación por diversos motivos. Un fenómeno puede tener múltiples explicaciones (Ault 1998), por lo que el uso de pruebas será determinante para descartar unas frente a otras. Además, algunos contextos y contenidos geológicos permiten abordar controversias socio-científicas (Trend 2009), cercanas en lo físico y lo social y emocional al alumnado, donde se le anime a contrastar opiniones basadas en evidencias a las que puede acceder.

\section{Modelización}

Los modelos científicos se entienden como representaciones abstractas que, simplificando la realidad y centrándose en ciertas características clave, tratan de explicar y predecir fenómenos científicos (Schwarz et al. 2009). Un modelo es, por tanto, una representación parcial que muestra ciertos aspectos del fenómeno con la finalidad específica de desarrollar explicaciones acerca de éste (Gilbert et al. 2000). El desarrollo de modelos científicos escolares comprende de operaciones como la expresión del modelo, hacer analogías o realizar abstracciones, evaluar y revisar el modelo o aplicar y generalizar el modelo a otras situaciones (Justi y Gilbert 2002). En el proceso de modelización de sistemas geológicos es muy recomendable el uso de recursos como la 
realización de analogías, la construcción de maquetas (Gray et al. 2011, Martínez et al. 2016) o los dibujos y diagramas donde representar los elementos dinámicos y el funcionamiento del sistema (Garrido 2016, Justi y Gilbert 2002, Márquez y Artes 2016). Este tipo de sistemas de expresión, junto con la asistencia guiada del docente en forma de preguntas clave o scaffoldings (Domènech 2015), permite una continua reestructuración de ideas, una de las operaciones clave en la modelización.

\section{MÉTodo}

La metodología de investigación de este trabajo es el análisis de contenido (López 2002) de documentos publicados en revistas científicas que se enmarca en la metodología cualitativa (Bowen 2009). En este caso, la búsqueda se realizó en una selección de revistas, en un rango temporal. Así, siguiendo las tres fases (lectura superficial, lectura e interpretación) que implica el análisis de documentos (Bowen 2009) se procedió a identificar trabajos que contenían los términos de búsqueda (lectura superficial) que se indican más adelante, a seleccionar experiencias didácticas que cumplían con los criterios (lectura), y finalmente, a describir el contenido de los artículos en relación con las preguntas de investigación (interpretación).

El proceso de selección de artículos se resume en el diagrama de la Figura 1, adaptado de la guía PRISMA para la revisión sistemática de trabajos (BMJ 2021). Primeramente, se buscaron artículos que abordaran las salidas de campo en una selección de cinco revistas estatales y cinco internacionales entre las más relevantes del área de Didáctica de las Ciencias (Tabla 1). De ellas dos son específicas de Didáctica de la Geología.

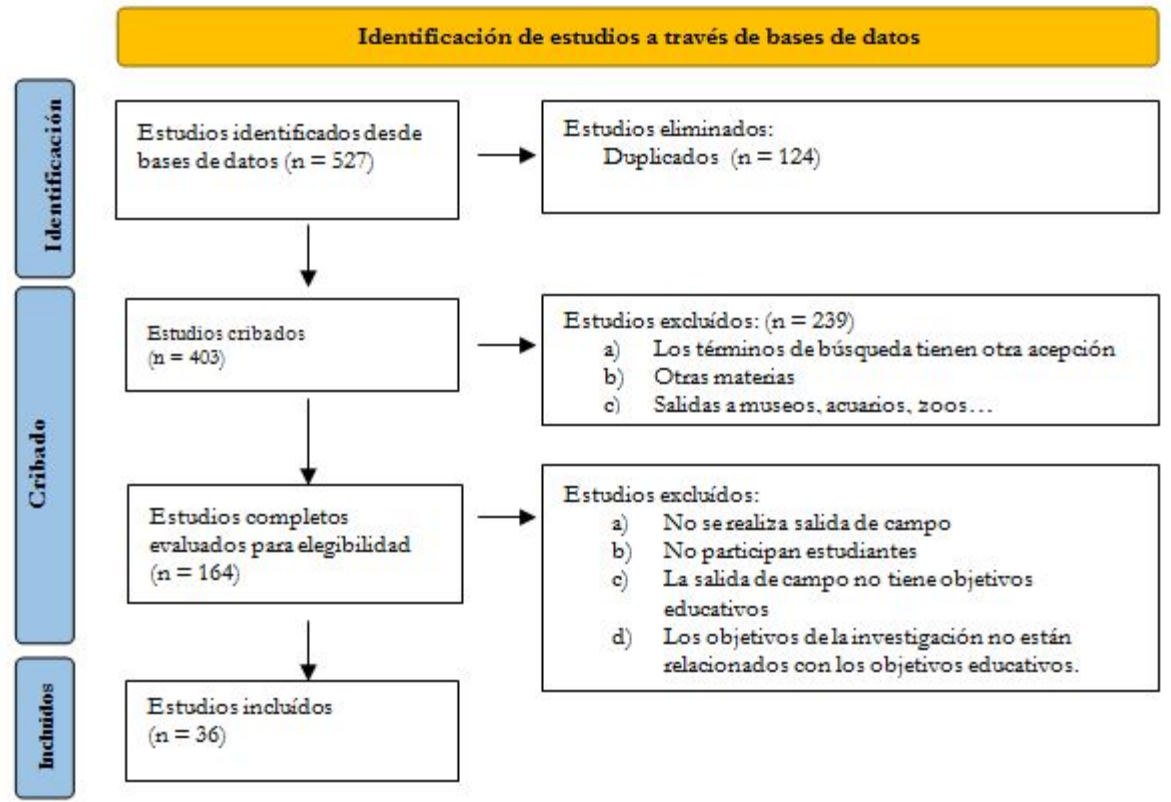

FIGURA 1

Resumen del proceso de identificación y cribado de estudios.

Diagrama adaptado de la propuesta PRISMA (BMJ 2021).

La búsqueda se hizo a través de bases de datos seleccionando el rango temporal 2011-2020 y limitando la búsqueda a las revistas mencionadas. Se buscaron los términos "salida", "trabajo de campo", "itinerario", "campo" para las revistas en castellano y "trip", "field work", "itinerary", "outside”, "outdoor” y "land" para las revistas en inglés en el título y resumen de los artículos. Se localizaron 403 trabajos en los que se mencionaran esos términos (después de eliminar los duplicados). Tras una lectura superficial, se excluyeron aquellos en los que los términos tenían otras acepciones (p. ejemplo "campo magnético terrestre") y los artículos sobre materias alejadas de las ciencias de la Tierra. Tampoco se consideraron las salidas a equipamientos tales como 
museos, acuarios, zoos u otro tipo de equipamientos similares, ya que el campo se ciñe más al ámbito de trabajo geológico (Pedrinaci 2012; Mogk y Goodwin 2012; Petcovic et al. 2014). Finalmente se seleccionaron 164 artículos para una lectura completa (tabla 1 ).

Estos artículos fueron revisados por pares entre los autores y se clasificaron como experiencias geológicas con salida (EGS) aquellos estudios que cumplían cuatro criterios: a) que hubieran realizado una salida de campo; b) que en ella hubieran tomado parte estudiantes (desde infantil hasta profesorado); c) que la salida tuviera objetivos educativos (implícitos o explícitos) relacionados con la geología; d) que los objetivos de la investigación estuvieran relacionados con los objetivos educativos. Aquellos en los que no hubo acuerdo inicial (15\%), fueron reevaluados por los tres, llegando a consensos en todos los casos. Finalmente, se categorizaron como EGS 36 trabajos (tabla 1). Las referencias de las 36 EGS pueden hallarse en el Anexo 1.

TABLA 1

Selección de experiencias.

\begin{tabular}{|c|c|c|c|}
\hline Revista & $\begin{array}{c}\mathrm{N}^{\circ} \text { artículos } \\
\text { con salida }\end{array}$ & $\mathrm{N}^{\circ}$ EGS & Códigos de EGS \\
\hline Alambique & 7 & 0 & \\
\hline Enseñanza de las Ciencias & 2 & 0 & \\
\hline Enseñanza de las Ciencias de la Tierra & 38 & 8 & EGS01-EGS08 \\
\hline International Journal of Science Education & 18 & 0 & \\
\hline Journal of Geoscience Education & 48 & 23 & EGS09-EGS31 \\
\hline Journal of Research in Science Teaching & 6 & 1 & EGS32 \\
\hline Research in Science Education & 22 & 2 & EGS33, EGS34 \\
\hline Revista Electrónica de la Enseñanza de las Ciencias & 3 & 0 & \\
\hline Revista Eureka sobre Enseñanza y Divulgación de las Ciencias & 7 & 1 & EGS35 \\
\hline \multirow[t]{2}{*}{ Science Education } & 13 & 1 & EGS36 \\
\hline & 164 & 36 & \\
\hline
\end{tabular}

Las 36 EGS fueron analizadas por los tres autores. Para determinar la alusión al desarrollo de prácticas científicas, se elaboró un listado de operaciones (tabla 2) que según los referentes teóricos se incluyen en las prácticas de indagación (Bargiela et al. 2018, Ferrés et al. 2015, Harlen 2014, Jiménez-Aleixandre y Crujeiras 2017, NRC 2012, Pedaste et al. 2015, Schalk et al. 2013), argumentación (Bargiela et al. 2018, Chang y Chiu 2008, Erduran et al. 2004, Jiménez-Aleixandre 2010, Jiménez-Aleixandre y Crujeiras 2017, NRC 2012, Osborne et al. 2016) y modelización (Bargiela et al. 2018, Garrido 2016, Gilbert et al. 2000, JiménezAleixandre y Crujeiras 2017, NRC 2012, Schwarz et al. 2009). 
TABLA 2

Operaciones relacionadas con las prácticas científicas.

\begin{tabular}{|c|c|}
\hline Práctica científica & Operaciones \\
\hline \multirow{8}{*}{ Indagación } & IND1-Observar \\
\hline & IND2-Formular preguntas investigables \\
\hline & IND3-Formular hipótesis y/o predicciones \\
\hline & IND4-Identificar variables \\
\hline & IND5-Controlar variables \\
\hline & IND6-Planificar la investigación \\
\hline & IND7-Recoger datos \\
\hline & IND8-Analizar datos \\
\hline \multirow{7}{*}{ Argumentación } & ARG1-Identificar elementos del argumento \\
\hline & ARG2-Formular conclusiones \\
\hline & ARG3-Interpretar pruebas \\
\hline & ARG4-Usar pruebas \\
\hline & ARG5-Elaborar justificaciones \\
\hline & ARG6-Generar contraargumentos \\
\hline & ARG7-Evaluar argumentos \\
\hline \multirow{9}{*}{ Modelización } & MOD1-Revisar conocimientos previos \\
\hline & MOD2-Hacer analogías \\
\hline & MOD3-Realizar abstracciones \\
\hline & MOD4-Seleccionar el modo de representación \\
\hline & MOD5-Expresar/representar el modelo \\
\hline & MOD6-Evaluar el modelo \\
\hline & MOD7-Revisar el modelo \\
\hline & MOD8-Aplicar el modelo \\
\hline & MOD9-Explicar fenómenos naturales \\
\hline
\end{tabular}

Los autores identificaron la presencia de tales operaciones en la descripción que en los artículos se realizaba de las actividades realizadas por los estudiantes tanto en la salida como en el aula.

\section{Resultados}

\section{Prácticas científicas en las EGS}

En 20 de las 36 EGS se identificaron menciones a más de 2 operaciones diferentes de una determinada práctica. Respecto a la edad de los participantes en las 20 EGS, un 55\% eran universitarios, un 30\% de educación secundaria, un $10 \%$ profesores y un $5 \%$ de educación primaria.

En la Figura 2 se muestra qué prácticas fueron identificadas en las 20 EGS. 


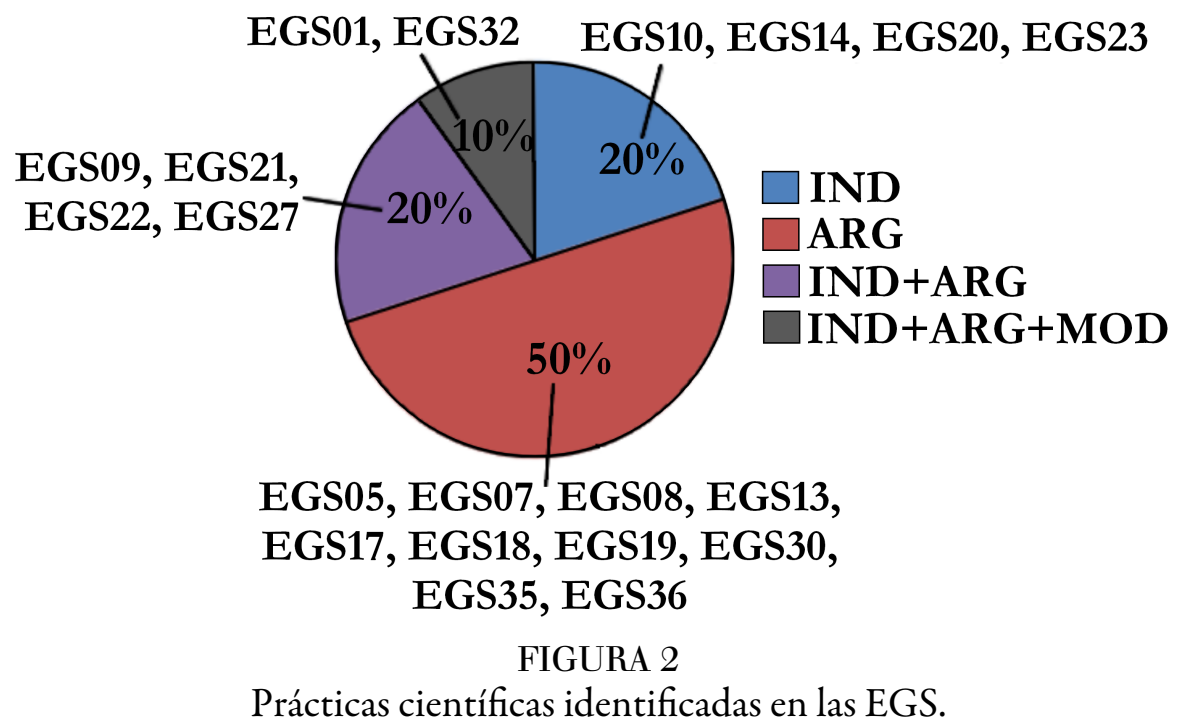

En el 50\% de las 20 EGS en que se identificaron prácticas científicas estaba presente la indagación, la argumentación en el $80 \%$ y la modelización únicamente en el 10\% que explicitaba operaciones relacionadas con las tres prácticas.

En la tabla 3 se muestra la proporción de EGS en que se identificaron prácticas en cada franja de edad.

TABLA 3

Porcentaje de EGS con prácticas científicas por cada franja de edad.

\begin{tabular}{ccccc}
\hline Edad* & Alguna práctica & IND & ARG & MOD \\
\hline 1 & 33 & 0 & 33 & 0 \\
2 & 50 & 8 & 42 & 0 \\
3 & 69 & 50 & 50 & 13 \\
4 & 40 & 20 & 40 & 0 \\
\hline
\end{tabular}

*1: Educación primaria; 2: Educación secundaria; 3: Estudios universitarios; 4: Profesorado.

Los resultados de la tabla 3 muestran que la proporción de EGS en las que se identificaron dos o más operaciones relacionadas con las prácticas científicas fue mayor cuanto mayor era la edad de los participantes en las experiencias, con la salvedad de las experiencias en que participó profesorado en formación. Esta relación fue más acusada en el caso de la indagación y la modelización, para las que se identificaron muy pocas experiencias fuera del ámbito universitario, pero con una relación más igualitaria en el caso de la argumentación. Respecto a las operaciones, algunas destacaron por la diferencia según la edad y en otras la edad pareció no influir. Así, IND7-Recoger datos fue la operación más mencionada en todos los grupos de edad sin apenas diferencias (entre un $67 \%$ y un $80 \%$ de estudios la citaron), mientras que en el caso de IND8-Analizar datos sí hubo diferencias por edad. Sin tener en cuenta las experiencias con profesorado, se hallaron correlaciones estadísticamente significativas entre la edad y la práctica de indagación $(\mathrm{r}=0,454$ significativa al 0,01), y la operación IND8-Analizar datos ( $\mathrm{r}=0,358$ significativa al 0,05$)$. En cuanto a las operaciones de argumentación, su mención fue bastante equilibrada (31-50\%) entre los grupos de edad en el caso de ARG2-Formular conclusiones, ARG3-Interpretar pruebas y ARG4-Utilizar pruebas; sin embargo se observó una diferencia no significativa según la edad $(0,17 \%, 25 \%, 40 \%$ respectivamente) para $A R G 5$ Elaborarjustificaciones. 


\section{Salida de campo respecto a aula}

Primeramente, se muestra en la figura 3 el número de EGS en las que se identificaron evidencias de realización de las prácticas científicas (más de dos operaciones diferentes), tanto en las actividades realizadas en el aula como en las realizadas en la salida, como en conjunto (en la salida, en el aula o en ambas).

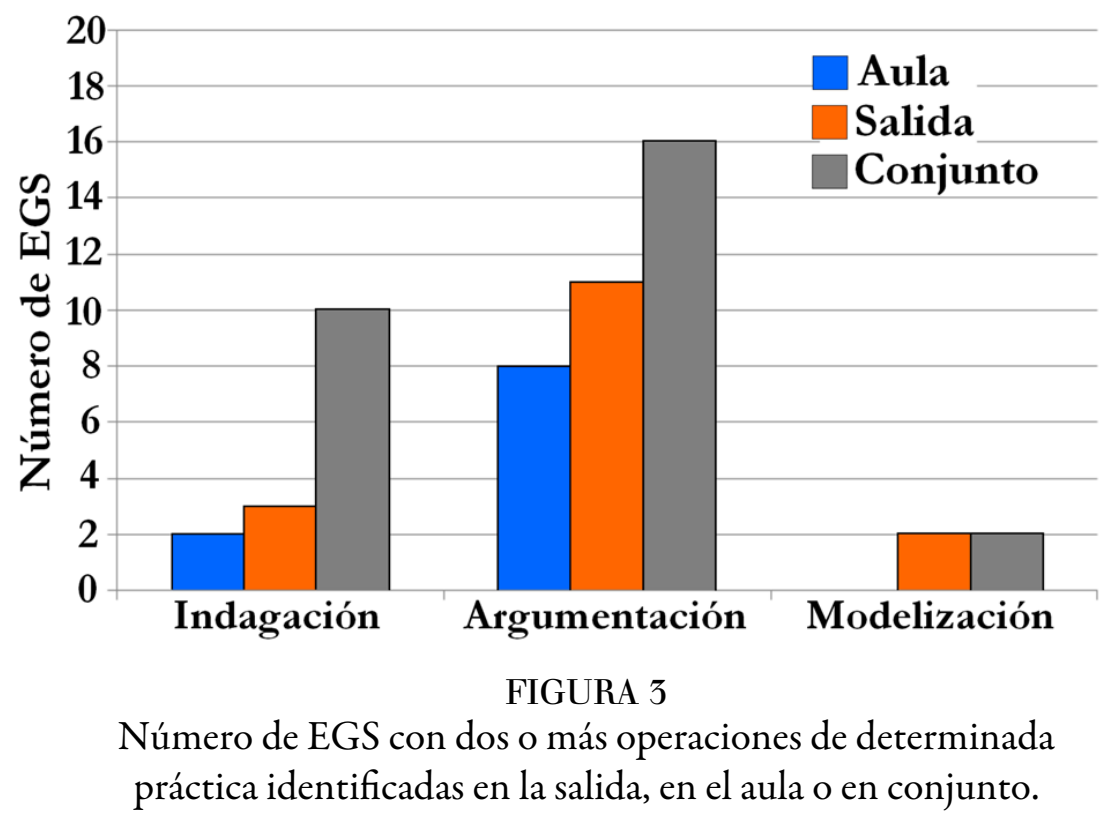

Como se observa en la figura 3, se identificaron más EGS en las que se explicitara la realización de más de dos operaciones de prácticas científicas en la salida que en el aula.

En la figura 4 se muestra el número de operaciones identificadas en cada EGS tanto en la salida como en el aula. Como se observa en la figura 4, la mayor parte de las EGS refirieron operaciones tanto en el aula como en la salida. De hecho, en 10 se mencionaron más en el aula que en la salida, en 9 más en la salida y en uno en igual número. Destaca que en 7 EGS se mencionaron más de cinco operaciones en la salida y en dos, más de cinco operaciones en el aula.

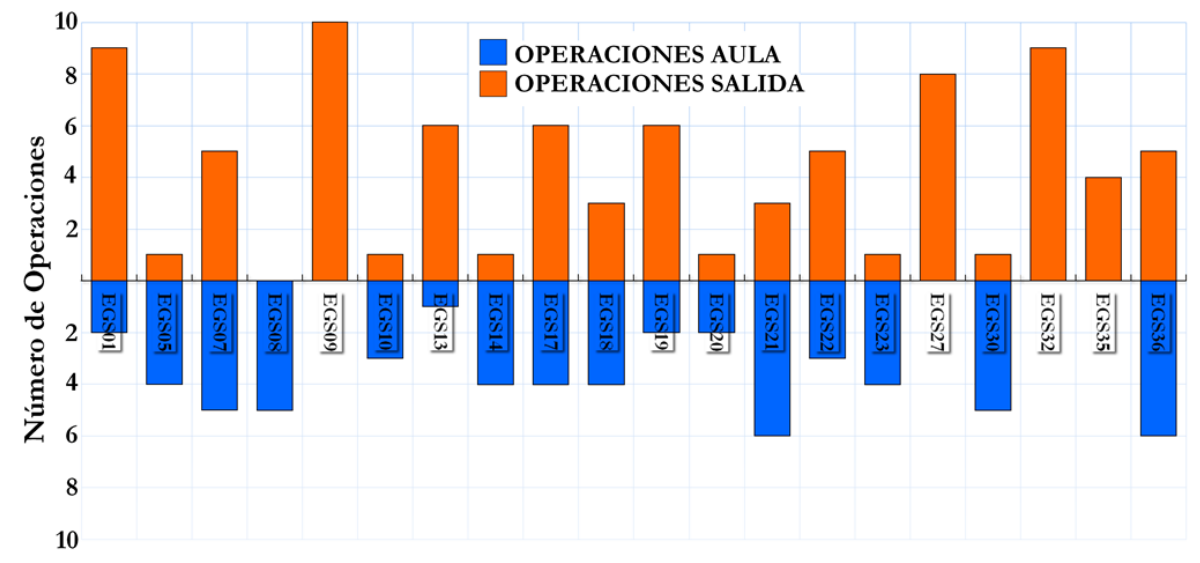

FIGURA 4

Número de operaciones de prácticas científicas identificadas en cada EGS tanto en la salida como en el aula.

En la figura 5 se muestran el número de EGS en los que se identificó la presencia de cada operación. 


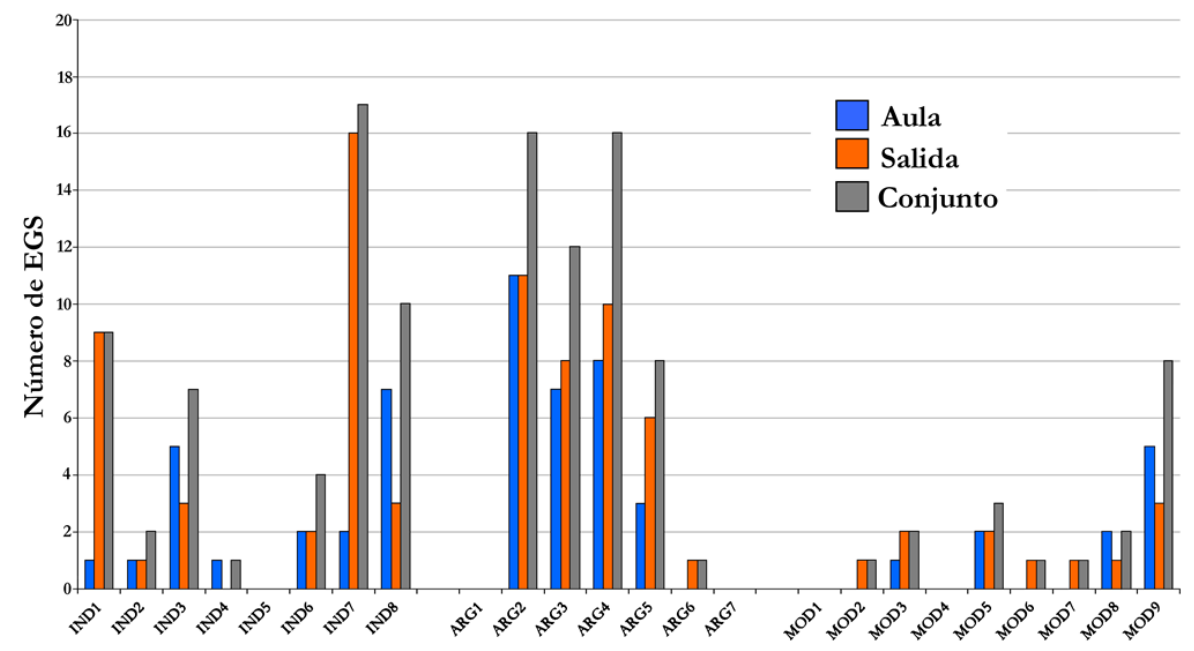

FIGURA 5

Número de EGS en las que identificó cada una de las operaciones.

Como se observa en la figura 5, 11 de las operaciones se mencionaron más al describir las actividades de la salida que las del aula, siendo la diferencia destacable en el caso de IND1-Observar e IND7-Recoger datos. 6 operaciones se explicitaron más en las actividades de aula, destacando IND8-Analizar datos.

\section{Operaciones de la indagación, argumentación y modelización}

Cuatro operaciones (IND7, ARG2, ARG3, ARG4) se mencionaron en la mitad o más de las 20 EGS (figura 5). Por otro lado, a 13 operaciones se aludió en dos o menos EGS. A continuación, se muestran los resultados desglosados por práctica científica.

\section{Práctica de indagación}

En la figura 6 se muestra el número de operaciones de indagación identificadas en cada EGS tanto en la salida como en el aula. 


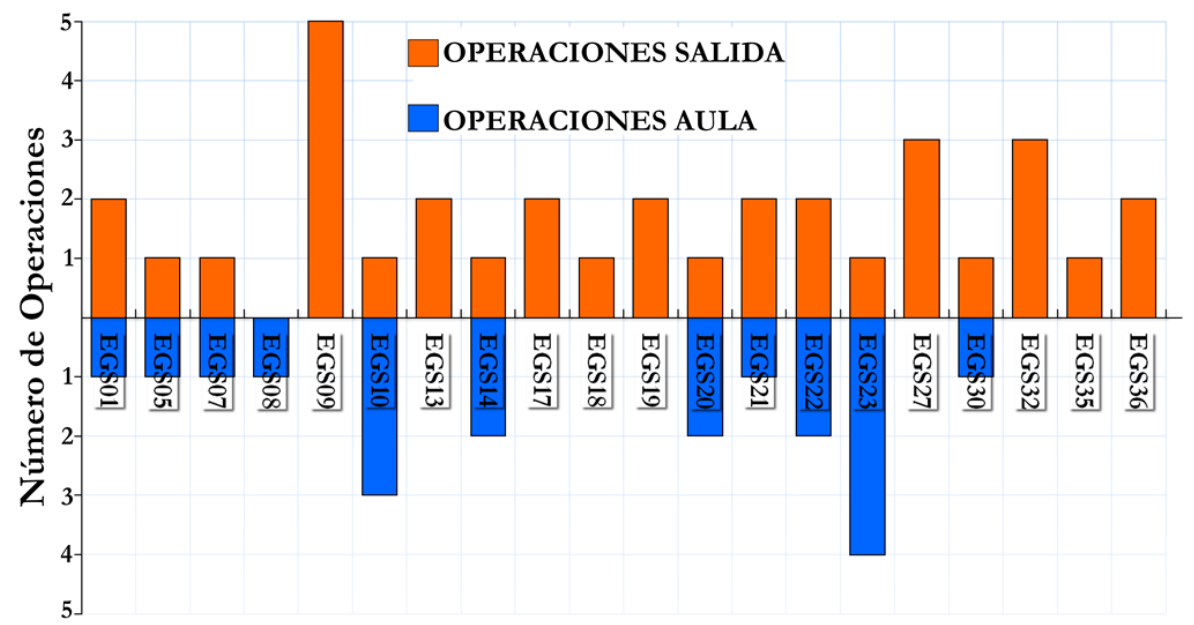

FIGURA 6

Número de operaciones de indagación identificadas en cada EGS tanto en la salida como en el aula.

Las 20 EGS mencionaron al menos alguna operación de indagación. La mitad de ellas combinaron la alusión a operaciones en las actividades de la salida y las del aula. Del resto, solo 9 las mencionaron en las de la salida y una en las del aula. En la tabla 4 se muestra un fragmento de EGS09, en la que más operaciones de indagación en la salida se identificaron. En EGS09 (Almquist et al. 2011), los participantes (profesores/ as) realizaron diversas operaciones relacionadas con la argumentación (que se muestran más adelante) y la indagación para interpretar el ambiente sedimentario en el que realizan la salida, que consta de varias paradas.

TABLA 4

Operaciones relacionadas con la indagación en EGS09.

\begin{tabular}{ll}
\hline \multicolumn{1}{c}{ Extracto de EGS09 (Almquist et al. 2011, pp. 34-35) } & Operaciones* \\
\hline En el campo (...) Antes de recoger datos, el grupo completo formuló una & IND3 $(s)$ \\
hipótesis para interpretar el ambiente sedimentario de Tullock y desarrolló & \\
un diseño experimental para testar la hipótesis. $(. .$.$) los equipos de$ & IND6 $(s)$ \\
estudiantes-profesor realizaron observaciones $(. .$.$) y recolectaron muestras$ & IND1 \\
& IND7 $(s)$ \\
de sedimentos para un posterior análisis en el laboratorio. & IND8 $(a)$ \\
\hline
\end{tabular}

* $s$ indica que se realiza en la salida, $a$, en el aula

Como se ha mencionado en el apartado anterior, dos de las operaciones de indagación destacaron por la diferencia de explicitación en las actividades de la salida frente a las de aula (véase figura 5). De hecho, 16 EGS declaraban IND7-Recoger datos en la salida y en 6 la única operación realizada en la salida ha sido la de IND1-Observar o IND7-Recoger datos.

La figura 5 muestra además que hay operaciones que apenas se mencionaron, entre ellas IND2-Formular preguntas investigables. 


\section{Práctica de argumentación}

En la figura 7 se muestra el número de operaciones de argumentación identificadas en cada EGS, tanto en la salida como en el aula.

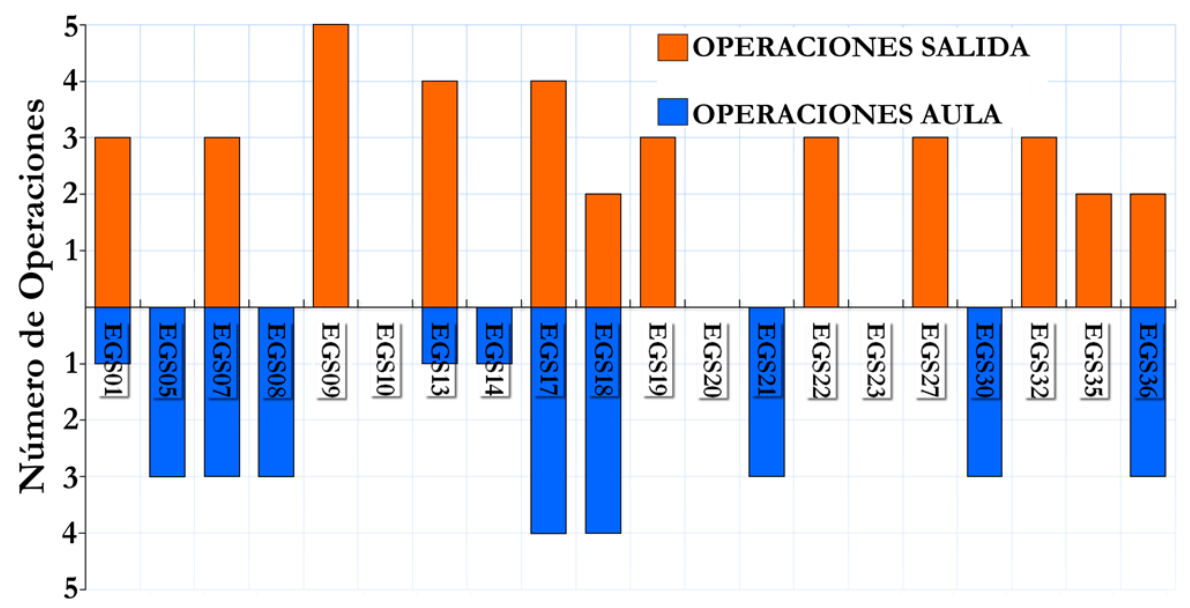

FIGURA 7

Número de operaciones de argumentación identificadas en cada EGS tanto en la salida como en el aula.

17 EGS mencionaron al menos alguna operación de argumentación. En 6 se explicitaron tanto en la descripción de la salida como en la de las actividades de aula. En 6 exclusivamente en la salida, y en 5 solo en el aula. En las tablas 5 y 6 se muestran fragmentos de EGS09 y EGS07. En EGS07 (Blanco-Ferrera et al. 2019) casi cien alumnos de $4^{\circ}$ de la Enseñanza Secundaria Obligatoria (ESO) y de la asignatura Geología de $2^{\circ}$ curso de Bachillerato realizaron actividades en el aula y en el campo (costa asturiana) para, a partir de fósiles del Cretácico, identificar los distintos medios sedimentarios registrados, e inferir las situaciones de transgresión y regresión del mar.

TABLA 5

Operaciones relacionadas con la argumentación en EGS09.

\begin{tabular}{ll}
\hline \multicolumn{1}{c}{ Extracto de EGS09 (Almquist et al. 2011, pp. 34-35) } & Operaciones* \\
\hline Los participantes utilizaron las pruebas de sedimentos que habían & ARG4 $(a)$ \\
recolectado $(. .$.$) para interpretar los cambios temporales en el ambiente.$ & \\
$\begin{array}{l}(. . .) \text { Se les dijo que compararan estas observaciones con las que habían } \\
\text { observado en la parada anterior, y que anotaran las diferencias. Los }\end{array}$ & ARG4 $(s)$ \\
instructores $(. .$.$) les solicitaron que utilizaran estas líneas de evidencias de$ & ARG3 $(s)$ \\
manera conjunta con su conocimiento sobre la historia geológica de la & ARG5 $(s)$ \\
región $(. .$.$) para inferir el ambiente deposicional (. .$.$) . Los participantes$ & ARG2 $(s)$ \\
habían alcanzado sus propias conclusiones. & \\
\hline
\end{tabular}

* $s$ indica que se realiza en la salida, $a$, en el aula 
TABLA 6

Operaciones relacionadas con la argumentación en EGS07.

\begin{tabular}{ll}
\hline \multicolumn{1}{c}{ Extracto de EGS07 (Blanco-Ferrera et al. 2019, p. 21) } & Operaciones* \\
\hline $\begin{array}{l}\text { Identificar y reconocer distintos tipos de fósiles cretácicos e interpretar el } \\
\text { medio ambiente en el que vivieron, de acuerdo con los datos }\end{array}$ & ARG3 $(a)$ \\
paleoecológicos ofrecidos en el taller. & ARG2 $(a)$, ARG4 $(a)$ \\
$(\ldots)$ & \\
- Reconocer los fósiles y los distintos tipos de rocas sedimentarias en los & ARG3 $(s)$ \\
que se encuentran. & ARG2 $(s)$ \\
- Interpretar el medio sedimentario en el que vivieron e indicarlo en la & \\
columna & \\
\hline & $s$ indica que se realiza en la salida, $a$, en el aula
\end{tabular}

En la figura 5 se observa que las operaciones mostradas en las tablas 5 y 6 son las que más se mencionaron. Sin embargo, hay otras operaciones, como ARG6-generar contraargumentos y ARG7-evaluar argumentos que apenas se identificaron.

\section{Práctica de modelización}

En la figura 8 se muestra el número de operaciones de modelización identificadas en cada EGS tanto en la salida como en el aula.

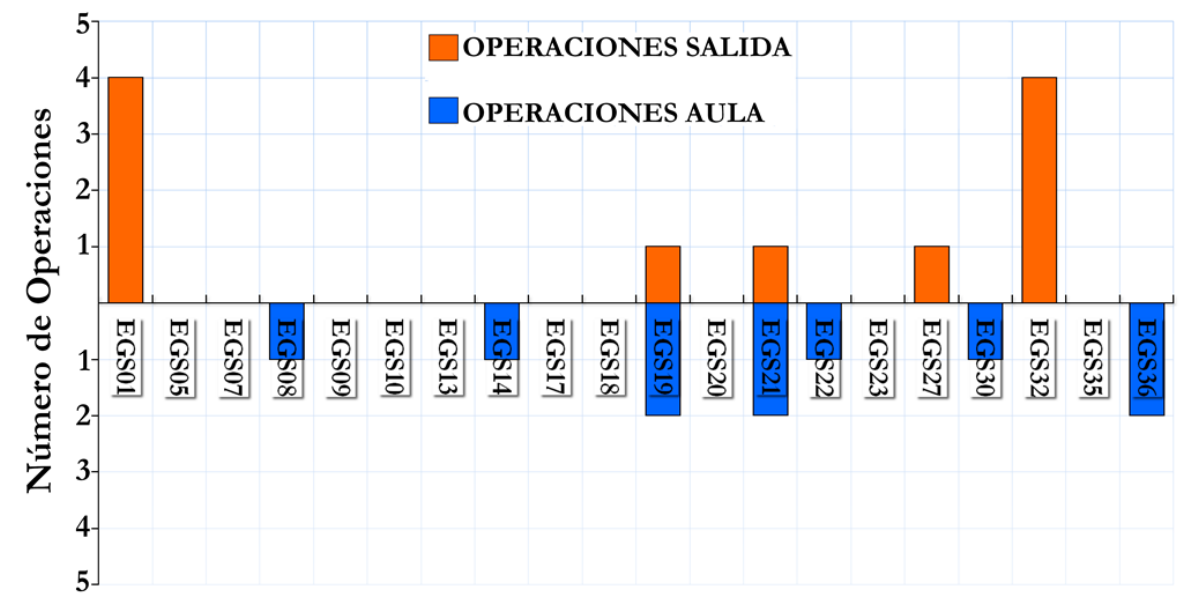

FIGURA 8

Número de operaciones de modelización identificadas en cada EGS tanto en la salida como en el aula.

Fueron solo 10 las EGS (la mitad) que mencionaron alguna operación relacionada con esta práctica. Dos EGS explicitaron operaciones de modelización tanto en la salida como en el aula. En EGS32, de la que se muestra un extracto en la tabla 7, estudiantes universitarios realizaron un examen individual en el campo, en el que debían construir el modelo geológico de una zona determinada (Balliet et al. 2015). 
TABLA 7

Operaciones relacionadas con la modelización en EGS32.

\begin{tabular}{ll}
\hline Extracto de EGS32 (Balliet et al. 2015, p. 1121) & Operaciones* \\
\hline Daniel afirma que durante su primer examen él tiene dos modelos: "Yo & \\
tuve dos modelos en marcha, tenía uno_era solo una sección recta, y una & \\
en la que ocurría algo raro"; este último se reveló como un modelo basado & MOD6 $(s)$ \\
en una unidad plegada de roca. El final de las notas de Daniel muestra un & \\
dibujo de un modelo completado que destaca el pliegue mencionado & MOD5 $(s)$ \\
anteriormente. Esto muestra que Daniel deja de lado el modelo sin pliegue & MOD7 $(s)$ \\
y se centra en la hipótesis del pliegue. & \\
$*$ * indica que se realiza en la salida, $a$, en el aula
\end{tabular}

Como se observa en la figura 5, solo se aludió de forma generalizada a la operación MOD9-explicar fenómenos naturales, y otras operaciones como la representación (MOD5), evaluación (MOD6) y revisión (MOD7) del modelo apenas se mencionaron.

\section{DISCUSIÓN, IMPLICACIONES Y CONCLUSIONES}

En este trabajo se ha abordado la presencia de prácticas científicas en las salidas de campo en geología, para lo que se identificaron las operaciones correspondientes en las propuestas educativas con contenido geológico que incluían salida de campo (EGS) aparecidas en los artículos publicados en los últimos 10 años en 10 revistas de didáctica de las ciencias.

Este estudio tiene limitaciones inherentes a la metodología de análisis de documentos (Bowen 2009). Un factor limitante viene dado por la muestra, dado que se partió de una selección de revistas de un rango temporal. Otro factor es lo que Bowen (2009, p. 31) denomina insuficiente detalle. Los datos para el análisis consistieron en las descripciones de las actividades narradas en los artículos. Es destacable que la referencia al desarrollo y/o al análisis de las prácticas en los objetivos de las EGS fue muy baja, lo que puede haber incidido en el escaso detalle a la hora de aludir a las operaciones. Así, es probable que en las secuencias se realizaran más operaciones relacionadas con las prácticas científicas que las halladas. Aún con esta limitación, los resultados del análisis llevan a poder formular algunas conclusiones.

Los resultados muestran que en 20 trabajos (el 56\% de las 36 EGS identificadas) se mencionó la realización de alguna práctica científica en el conjunto de la secuencia. Las tres prácticas se identificaron, destacando la alta presencia de la argumentación y la baja de la modelización. Se puede decir, por tanto, que una mayoría de las EGS se diseñaron en consonancia con las propuestas actuales de desarrollo de la competencia científica (OECD 2019) a través de la práctica científica (NRC 2012). Esto sitúa estas propuestas en línea con la perspectiva educativa del aprendizaje situado que busca la enculturación (Brown et al. 1989, Donaldson et al. 2020) de los estudiantes en una disciplina como parte de su aprendizaje, a través de la realización de actividades auténticas. Se apreciaron algunas diferencias según la edad de los participantes en las experiencias, lo que puede ser reflejo de que algunas operaciones relacionadas con las prácticas científicas suponen una alta demanda cognitiva para el alumnado de primaria y secundaria. Ello explicaría que la operación IND7-Recoger datos fuera realizada en todas las franjas de edad; pero IND8-Analizar datos presentara marcadas diferencias a favor de los más mayores. Otro ejemplo es $A R G 5$-Elaborarjustificaciones, para la que se hallaron diferencias por edad que se corresponden con las dificultades encontradas por Ryu y Sandoval (2012) en alumnado de 
primaria para elaborar justificaciones en comparación a utilizar pruebas. En las experiencias con profesorado se observa que la presencia de las prácticas fue menor que en el ámbito universitario, con un amplio margen de mejora. Esta baja presencia no se justifica por la demanda cognitiva, por lo que sería necesario aumentar las experiencias de este tipo con el profesorado con el fin de que éste las ponga en práctica con su alumnado.

Respecto a la segunda pregunta de investigación, los resultados muestran que en las secuencias analizadas la salida de campo fue un contexto utilizado, en algunos casos preferentemente al aula, para el desarrollo de las prácticas científicas. La operación mayormente realizada en la salida fue IND7-Recoger datos, que concuerda con la idea de que la esencia del trabajo de campo es la recolección de datos científicos fuera del aula o laboratorio (Balliet et al. 2015). Sin embargo, la presencia en la salida de otras operaciones de indagación, argumentación y en menor medida de modelización, hace ver que el trabajo de campo fue más allá de la recolección de muestras. De hecho, en prácticamente la mitad de EGS se mencionaron más operaciones relacionadas con las prácticas científicas en las actividades de la salida que en las del aula. Además, los resultados muestran que algunas operaciones además de la mencionada IND7-Recoger datos, por ejemplo, ARG2-Formular conclusiones, ARG3-Interpretar pruebas, ARG4-Usar pruebas y ARG5-Elaborar justificaciones, fueron realizadas fundamentalmente en la salida. Podría decirse, por tanto, que la salida jugó un papel educativo sustancial y no meramente ilustrativo, social o lúdico, en línea con lo reclamado desde la didáctica de las ciencias y de la geología en particular (Behrendt y Franklin 2014, Orion y Hofstein 1994, Pedrinaci 2012), dando a la salida ese papel fundamental que debe tener en geología (Egger 2019).

En lo referente al desarrollo de las distintas operaciones de cada práctica (tercera pregunta de investigación), los resultados son diferentes para las diversas operaciones y para las tres prácticas. En cuanto a la práctica de indagación, se podría decir que no se trabajó en todo su potencial, ya que salvo las operaciones IND1-Observar o IND7-Recoger datos, en pocas EGS se encontraron evidencias de otras operaciones que completaran el ciclo de indagación (Pedaste et al. 2015). En el caso de IND4-Identificar variables e IND5Controlar variables, su ausencia podría deberse a la propia naturaleza de la geología, ya que es una ciencia más interpretativa y quizás menos experimental que otras ciencias en el sentido estricto de manipular las variables para comprobar resultados empíricamente. Sin embargo, destaca la escasez en la que se alude a operaciones claves para la indagación como IND2-Formular preguntas investigables (Harlen 2014, Martí 2012, Sanmartí y Márquez 2012). Dado que en el 65\% de las 20 EGS los participantes eran adultos, se podría pensar que se trataba de participantes con los que se podían plantear trabajos de investigación de autonomía alta (Martí 2012) en el que ellos se apropiaran del proceso completo. Un buen ejemplo es EGS23 (Kelley et al. 2015) en que los 18 estudiantes universitarios, después de conocer el entorno, se cuestionan qué tipo de preguntas de investigación pueden plantear, formulan hipótesis, recogen datos que analizan. Los autores concluyeron que realizar el proceso completo desde la identificación de problemas que investigar dio a los estudiantes un sentido de apropiación del proyecto y que el campo y las situaciones influyeron en el aprendizaje de los estudiantes. Para mejorar el desarrollo de la indagación en secuencias con salida de campo, Abolins (2014) propuso incrementar las tareas investigativas abiertas fuera del aula de tal manera que los estudiantes trabajaran con distintas hipótesis a la hora de interpretar los fenómenos, de manera consecuente con la naturaleza interpretativa de la geología (Ault 1998), en la que la explicación o reconstrucción de un fenómeno puede tener múltiples explicaciones.

De los resultados puede deducirse que la práctica de argumentación fue una práctica bastante presente en las experiencias seleccionadas, ya que operaciones características de la práctica de argumentación, el interpretar datos (ARG3) para luego utilizarlos como pruebas (ARG4) y con ellas formular conclusiones (ARG2) y/o justificar afirmaciones (ARG5), se mencionaron en hasta 16 EGS. Esto concuerda con lo expuesto por Trend (2009) respecto a lo propicia que es la geología para la argumentación. Sin embargo, operaciones relacionadas con una alta capacidad argumentativa (Erduran et al. 2004, Osborne et al. 2016) no se mencionaron, por ejemplo, ARG6-generar contraargumentos y ARG7-evaluar argumentos. Para favorecer el desarrollo de estas operaciones sería necesario diseñar actividades que las fomenten, por ejemplo, 
discusiones en las que se puedan contrastar diferentes argumentos y que promuevan la formulación de refutaciones (Jiménez-Aleixandre 2010). Para ello se puede aprovechar el carácter multicausal de la geología (Trend 2009) o tratar problemas o temas sociocientíficos relacionados con la zona visitada. En este último sentido, por ejemplo, en la EGS05 (Míguez-Rodríguez y González 2017) visitaron una explotación de pizarra para luego realizar un debate sobre conservación y progreso, analizando las ventajas y los inconvenientes de las diferentes opciones. Las discusiones sobre la suficiencia y validez de las pruebas también pueden ser una actividad a introducir para mejorar el desempeño en argumentación. Actividades de este tipo fueron identificadas en EGS22 (Soja 2014) y en discusiones facilitadas por los instructores a lo largo de la salida en EGS09 (Almquist et al. 2011). En este último, los autores concluyeron que la discusión sobre la validez de las pruebas de sus argumentos «ayudó a los participantes a construir argumentos científicos válidos, y a basar sus argumentos en pruebas bien examinadas y razonamientos concretos» (p. 39).

Por último, en lo que respecta a la práctica de modelización, solo se aludió de forma generalizada a la operación MOD9-explicar fenómenos naturales, mientras que otras operaciones importantes (Schwarz et al. 2009), como la representación (MOD5), evaluación (MOD6), revisión (MOD7) y aplicación (MOD8) del modelo apenas se mencionaron. El hecho de que, a diferencia de la indagación y la argumentación, en la modelización no se mencionen siquiera las operaciones básicas, sitúa a esta práctica en una posición inferior. Esto es un problema ya que la modelización puede ser una de las prácticas más útiles a la hora de aprender a mirar e interpretar los fenómenos geológicos (Kali et al. 2003). Se podría comenzar a trabajar la modelización con representaciones visuales como, por ejemplo, dibujos (Martínez-Peña y Gil-Quílez 2014) y maquetas (Gray et al. 2011, Nebot 2020), que en el caso específico de la geología ayudan no solo a representar las estructuras y los elementos dinámicos del modelo, sino también a dimensionar éstos y a trabajar la visión espacial. Así, también en el caso de la modelización, la salida de campo podría jugar un papel primordial, ya que el campo ofrece una oportunidad para revisar, evaluar y aplicar el modelo en un contexto real (Pedrinaci 2012).

A modo de conclusión final, se puede afirmar que las secuencias analizadas están en línea con la perspectiva de realizar actividades auténticas que incluyen prácticas científicas, y que además hicieron uso para ello de un contexto tan auténtico para la práctica de la actividad geológica como es el campo. De todas formas, y como se ha discutido, hay margen para que en secuencias educativas que se diseñen en el futuro puedan incrementarse tanto la presencia de determinadas operaciones como el papel de la salida de campo en su desarrollo. Para ello, el profesorado será una clave importante y su formación deberá abordar el diseño de salidas de campo para ser utilizadas como contextos de práctica auténtica de la geología.

\section{Materiales SUPlementarios}

Anexo I (pdf)

\section{Agradecimientos}

Este trabajo ha sido financiado por la Ayuda a la Investigación: Mod. II Grupos de la UPV/EHU (códigos GIU19/008 y PPGA20/14).

\section{REFERENCIAS}

Abolins M. (2014) Undergraduates discovering folds in "flat" strata: An unusual undergraduate geology field methods. Journal of Geoscience Education 62 (2), 264-277. 
Araitz Uskola, et al. Revisión de experiencias sobre prácticas científicas en Secuencias educativa...

Aguilera D. (2018) La salida de campo como recurso didáctico para enseñar ciencias. Una revisión sistemática. Revista Eureka sobre Enseñanza y Divulgación de las Ciencias 15 (3), 3103/1-3103/17.

Almquist H., Stanley G., Blank L., Hendrix M., Rosenblatt M., Hanfling S., Crews J. (2011) An integrated field-based approach to building teachers' geoscience skills. Journal of Geoscience Education 59, 31-40.

Apedoe X.S. (2008) Engaging students in inquiry: Tales from an undergraduate geology laboratory-based course. Science Education 92, 631-663.

Ault C.R. (1998) Criteria of excellence for geological inquiry: The necessity of ambiguity.Journal of Research in Science Teaching 35 (2), 189-212.

Balliet R., Riggs E., Maltese A. (2015) Students' problem solving approaches for developing geologic models in the field. Journal of Research in Science Teaching 52 (8), 1109-1131.

Bargiela I.M., Puig B., Blanco P. (2018) Las prácticas científicas en infantil. Una aproximación al análisis del currículum y planes de formación del profesorado de Galicia. Enseñanza de las Ciencias 36 (1), 7-23.

Behrendt M., Franklin T. (2014) A review of research on school field trips and their value in education. International Journal of Environmental \& Science Education 9, 235-245.

Blanco-Anaya P., Justi R., Díaz de Bustamante J. (2017) Challenges and opportunities in analysing students modelling. International Journal of Science Education 39 (3), 377-402.

Blanco-Ferrera S., Sanz-López J., Domínguez-Cuesta M.J., López-Fernández C., Pando L.A., Martos E. (2019) Transgresiones, regresiones y fósiles. Enseñanza de las Ciencias de la Tierra 27 (1), 18-30.

BMJ (2021) The PRISMA 2020 statement: an updated guideline for reporting systematic reviews. Research Methods \& Reporting 372(n71). https://doi.org/10.1136/bmj.n71

Bowen G.A. (2009) Document analysis as a qualitative research method. Qualitative Research Journal 9 (2), 27-40.

Brown J.S., Collins A., Duguid P. (1989) Situated cognition and the culture of learning. Educational Researcher 18 (1), 32-42.

Carcavilla L., Berrio M.P., Belmonte A., Durán J.J., López-Martínez J. (2010) Geological diffusion among the general public: principles, techniques and methods for the design of written information. Boletin de la Real Sociedad Española de Historia Natural (Sección geológica) 104, 93-110.

Carrier S.J., Tugurian L.P., Thomson M.M. (2013) Elementary science indoors and out: Teachers, time, and testing. Research in Science Education 43, 2059-2083. doi:10.1007/s11165-012-9347-5

Chang S.N., Chiu M.H. (2008) Lakatos' scientific research programmes as a framework for analysing informal argumentation about socio - scientific issues. International Journal of Science Education 30 (13), 1753-1773. https://doi.org/10.1080/09500690701534582

COSCE (2011) Informe ENCIENDE. Enseñanza de las Ciencias en la Didáctica escolar para edades tempranas en España. Madrid. http://www.cosce.org/pdf/Informe_ENCIENDE.pdf

Costillo E., Borrachero A.B., Esteban R., Sánchez-Martín J. (2014) Aportaciones de las salidas al medio natural como actividades de enseñanza y de aprendizaje según profesores en formación. Indagatio Didáctica 6, 10-22.

DeWitt J., Storksdieck M. (2008) A short review of school field trips: Key findings from the past and implications for the future. Visitor Studies 11 (2), 181-197. https://doi.org/10.1080/10645570802355562

Domènech J. (2015) Una secuencia didáctica de modelización, indagación y creación del conocimiento científico en torno a la deriva continental y la tectónica de placas. Revista Eureka sobre Enseñanza y Divulgación de las Ciencias 12(1), 186-197.

Donaldson T., Fore G.A., Filippelli G.M., Hess J.L. (2020) A systematic review of the literature on situated learning in the geosciences: beyond the classroom. International Journal of Science Education 42 (5), 722-743. https://d oi.org/10.1080/09500693.2020.1727060

Egger A. (2019) The field as touchstone. Journal of Geoscience Education 67 (2), 97-99. https://doi.org/10.1080/10 899995.2019 .1596461

Erduran S., Simon S., Osborne J. (2004) TAPping into argumentation: Developments in the application of Toulmin's Argument Pattern for studying science discourse. Science Education 88 (6), 915-933. 
Fedesco H., Cavin D., Henares R. (2020) Field-based Learning in Higher Education. Journal of the Scholarship of Teaching and Learning 20 (1), 65-84.

Ferrés C., Marbá A., Sanmartí N. (2015) Trabajos de indagación de los alumnos: instrumentos de evaluación e identificación de dificultades. Revista Eureka sobre Enseñanza y Divulgación de las Ciencias 12 (1), 22-37.

Frodeman R. (1995) Geological reasoning: geology as an interpretive and historical science. Geological Society of America Bulletin 107 (8), 960-968.

Garrido A. (2016) Modelitzazió i models en la formació inicial de Mestres de primària des de la perspectiva de la pràctica cientifica (Tesis doctoral). Universitat Autònoma de Barcelona, Bellaterra.

Gilbert J.K., Boulter C.J., Elmer R. (2000) Positioning models in science education and in design and technology education. En J.K. Gilbert y C.J. Boulter (Eds.), Developing models in science education (pp. 3-17). Kluwer Academic Publisher.

Gray K.R., Owens K.D., Steer D.N., McConnell D.A., Knight C.C. (2011) An exploratory study using hands - on physical models in a large introductory. Earth science classroom: Student attitudes and lessons learned. Electronic Journal of Science Education 12 (2), 1-23. http://ejse.southwestern.edu/article/view/7391

Harlen W. (2014) Helping children's development of inquiry skills. Inquiry in Primary Science Education 1, 5-19.

Jiménez-Aleixandre M.P. (2010) 10 ideas clave. Competencias en argumentación y uso de pruebas. Grao\#.

Jiménez-Aleixandre M.P., Crujeiras B. (2017) Epistemic practices and scientific practices in Science education. En K.S. Taber y B. Akpan (Eds.), Science Education. New Directions in Mathematics and Science Education (pp. 69-80). Sense Publishers.

Justi R.S., Gilbert J.K. (2002) Modelling, teachers' views on the nature of modelling, and implications for the education of modellers. International Journal of Science Education 24 (4), 369-387.

Kali Y., Orion N., Eylon B.-S. (2003) Effect of knowledge integration activities on students' perception of the Earth's crust as a cyclic system. Journal of Research in Science Teaching 40(6), 545-565.

Kelley D.F., Sumrall J.L., Sumral J.B. (2015) Student-designed mapping project as part of a geology field camp. Journal of Geoscience Education 63 (3), 198-209.

Lavie N., Tal T. (2017) Field trips to natural environments: how outdoor educators use the physical environment. Communication and Public Engagement 7, 237-252.

López F. (2002) El análisis de contenido como método de investigación. XXI, Revista de Educación 4, 167-179.

Márquez C., Artés M. (2016) Propuesta de análisis de representaciones sobre el modelo de cambio geológico del alumnado del grado de educación primaria. Enseñanza de las Ciencias de la Tierra 24 (2), 169-181.

Martí J. (2012) Aprender ciencias en la educación primaria. Graó.

Martínez M.B., Gil M.J., de la Gándara M. (2016) Aportación de las experiencias a la construcción de modelos: el suelo como sistema. Enseñanza de las Ciencias de la Tierra 24 (2), 182-189.

Martínez-Peña M.B., Gil-Quílez M.J. (2014) Drawings as a tool for understanding geology in the Environment. Journal of Geoscience Education 62 (4), 701-713.

Míguez-Rodríguez L.J., González C. (2017) La explotación de la pizarra como contexto de aprendizaje para ayudar al alumnado de bachillerato a entender las relaciones entre minería y sociedad. Enseñanza de las Ciencias de la Tierra 25 (2), 203-212.

Mogk D.W., Goodwin C. (2012) Learning in the field: Synthesis of research on thinking and learning in the geosciences. Special Paper of the Geological Society of America 486, 131-163.

Moss E., Cervato C. (2016) Quantifying the level of inquiry in a reformed introductory geology lab course. Journal of Geoscience Education 64, 125-137.

National Research Council (2012) A framework for K-12 Science Education: practices, crosscuttingconcepts and core ideas. National Academy Press. https://doi.org/10.17226/13165

Nebot M.R. (2020) Modelización del relieve kárstico en el laboratorio. Alambique 102, 38-46.

OECD (2019) PISA 2018 Assessment and Analytical Framework. OECD Publishing https://doi.org/10.1787/b25e fab8-en 
Araitz Uskola, et al. Revisión de experiencias sobre prácticas científicas en Secuencias educativa...

Orion N., Hofstein A. (1994) Factors that influence learning during a scientific field trip in a natural environment. Journal of Research in Science Teaching 31 (10), 1097-1119.

Osborne J. (2014) Teaching scientific practices: Meeting the challenge of change.Journal of Science Teacher Education 25, 177-196.

Osborne J., Dillon J. (2008) Science Education in Europe: Critical Reflections. Nuffield Foundation.

Osborne J.F., Henderson J.B., MacPherson A., Szu E., Wild A., Yao S.Y. (2016) The development and validation of a learning progression for argumentation in science. Journal of Research in Science Teaching 53 (6), 821-846.

Pedaste M., Mäeots M., Siiman L.A., de Jong T., van Riesen S.A.N., Kamp E.T., ...Tsourlidaki E. (2015) Phases of inquiry-based learning: Definitions and the inquiry cycle. Educational Research Review 14, 47-61.

Pedrinaci E. (2012) Trabajo de campo y aprendizaje de las ciencias. Alambique 71, 81-89.

Petcovic H.L., Stokes A., Caulkins J.L. (2014) Geoscientists' perceptions of the value of undergraduate field education. GSA Today 24(7), 4-10.

Rickinson M., Dillon J., Teamey K., Morris M., Choi M.Y., Sanders D., Benefield P. (2004) A review of research on outdoor learning. National Foundation for Educational Research and King's College.

Ryu S., Sandoval, W. A. (2012) Improvements to elementary children's epistemic understanding from sustained argumentation. Science Education 96, 488-526.

Sanmartí N., Márquez C. (2012) Enseñar a plantear preguntas investigables. Alambique 70, 27-36.

Schalk H.H., van der Schee J.A., Boersma K.Th. (2013) The development of understanding of evidence in preuniversity biology education in the Netherlands. Research in Science Education 43, 551-578.

Schwarz C.V., Reiser B.J., Davis E.A., Kenyon L., Achér A., Fortus D., Shwartz Y., Hug B., Krajcik J. (2009) Developing a learning progression for scientific modeling: making scientific modeling accesible and meaningful for learners. Journal of Research in Science Teaching 46 (6), 632-654.

Soja C.M. (2014) A field-based biomimicry exercise helps students discover connections among biodiversity, form and function, and species conservation during Earth's sixth extinction. Journal of Geoscience Education 62 (4), 679-690.

Trend R. (2009) Commentary: Fostering students' argumentation skills in geoscience education. Journal of Geoscience Education 57 (4), 224-232.

van Loon A.J. (2008) Geological education of the future. Earth-Science Reviews 86, 247-254.

\section{INFORMACIÓN ADICIONAL}

Para citar este artículo: Uskola A., Seijas N., Sanz J. (2022) Revisión de experiencias sobre prácticas científicas en secuencias educativas de geología con trabajo de campo. Revista Eureka sobre Enseñanza y Divulgación de las Ciencias 19(1), 1105. doi: 10.25267/Rev_Eureka_ensen_divulg_cienc.2022.v19.i1.1105 